\title{
Target Tracking in Boost Stage with Quantized Measurements of Space-based Infrared Cameras*
}

\author{
Yu WANG, Xiaogang WANG, ${ }^{\dagger}$ and Naigang CUI \\ School of Astronautics, Harbin Institute of Technology, Harbin 150001, China
}

\begin{abstract}
A novel state estimation method is proposed for target tracking in the boost stage using space-based infrared cameras (SBIRC) whose measurements are essentially corrupted by both Gaussian noise and quantization noise. As the quantization noise has non-Gaussian properties, conventional extended Kalman filtering (EKF) suffers from poor performance. The quantization noise of SBIRC is modelled using the mid-riser quantizer, which is usually adopted in the digital signal processing field. A novel minimum square error (MMSE) state estimation algorithm with quantized measurements, named the quantized extended Kalman filtering (QEKF), is then proposed. The time update is given based on first-order linearization of the nonlinearities, and the measurement update is derived based on the conditional mean estimate given the quantized measurements. As the multidimensional integrals in the measurement update derived doesn't have analytical solutions, a numerical integration method is proposed by combining Genz's transformation and quasi-Monte Carlo (QMC) method, which can avoid the curse of dimensionality. To further improve the tracking accuracy, quantized high-degree cubature Kalman filtering (QHCKF) is developed by integrating the fifth-degree cubature rule into the framework of the QEKF. Numerical simulation results illustrate the superiority of the proposed QEKF and QHCKF methods.
\end{abstract}

Key Words: Target Tracking, Quantized Measurements, Space-based Infrared Cameras, Spacecraft

\section{Nomenclature}

$\alpha$ : magnitude of thrust and drag net acceleration

$\beta$ : auxiliary variable

$\omega_{e}$ : Earth rotation rate

$\omega_{i}$ : weights of cubature points

$\mu$ : gravitational parameter

$\varepsilon_{y}, \varepsilon_{z}$ : length of the pixel in the direction of $\boldsymbol{y}_{p}$ and $z_{p}$

$w_{y}, w_{z}$ : zero-mean Gaussian white noise in the direction of $y$ and $z$

$\chi_{k \mid k-1, i}$ : transformed cubature points from the state model

$\Upsilon_{k \mid k-1, i}:$ transformed cubature points from the measurement model

$\Delta$ : length of the quantization interval

$a$ : target acceleration during the boost phase

$\boldsymbol{a}_{G}$ : acceleration of gravity

$\boldsymbol{a}_{N}$ : net acceleration of the thrust and drag

$A$ : quantization interval

$C_{e}^{b}$ : transformation matrix from the ECEF-CS to the body coordinate system

$e_{y}, e_{z}:$ quantization noise in the direction of $y$ and $z$

$f$ : focal length of SBIRC

$f$ : nonlinear state function

$\boldsymbol{F}_{k-1}$ : Jacobian of the vector $\boldsymbol{f}$

$\boldsymbol{h}$ : nonlinear measurement function

$\boldsymbol{H}_{k}$ : Jacobian of the measurement function $\boldsymbol{h}$

$\boldsymbol{K}_{k}$ : filter gain

C 2020 The Japan Society for Aeronautical and Space Sciences

*Received 17 July 2019; final revision received 10 January 2020; accepted for publication 18 March 2020.

†Corresponding author, wangxiaogang@hit.edu.cn $m$ : target mass

$\boldsymbol{P}_{k \mid k-1}$ : state prediction covariance

$Q$ : mid-riser quantizer

$r$ : dimension of $\boldsymbol{y}_{k}$

$S_{k}$ : innovation covariance

$v_{r}:$ target speed

$[x, y, z]$ : coordinate of the target in ECI-CS

$x$ : state vector

$\hat{\boldsymbol{x}}_{k \mid k-1}$ : predicted state

$\boldsymbol{y}_{k}^{i}$ : ideal measurement of the $i$ th satellite at time $k$

$\hat{\boldsymbol{y}}_{k}$ : predicted measurement

$\boldsymbol{y}_{k}^{q}:$ measurement vector after quantization

$\left(y_{p}, z_{p}\right)$ : ideal coordinates of the target in the pixel coordinate system

$\left(y_{p}^{q}, z_{p}^{q}\right)$ : coordinates of the target in the pixel coordinate system

$\boldsymbol{Y}_{k}^{q}$ : sequence of quantized measurements

AUN: additive uniform noise

DSP: digital signal processing

ECEF-CS: Earth center Earth fixed coordinate system

ECI-CS: Earth center inertial coordinate system

EKF: extended Kalman filtering

IR: infrared cameras

MMSE: minimum square error

QEKF: quantized extended Kalman filtering

QHCKF: quantized high-degree cubature Kalman filtering

QMC: quasi-Monte Carlo

RMSE: root-mean-square error

SBIRC: space-based infrared cameras

Subscripts

$k$ : time $k$

0 : initial 

$p$ : pixel coordinate
$b$ : body coordinate of satellites
$i$ : satellite index

\section{Introduction}

Space-based infrared cameras (SBIRCs) are commonly used for target tracking in the boost stage. ${ }^{1-5)}$ SBIRCs combine the advantages of space-based platform and optical sensor, passively recording target thermal radiation data to locate targets, which makes them more defensible. ${ }^{6)}$ A commonly employed strategy is to represent the statistical properties of the noise of infrared cameras (IR) by Gaussian distributions, ${ }^{7)}$ or mixture Gaussian distributions. ${ }^{8)}$ However, the image plane of SBIRCs is composed of discrete pixels, which leads to discontinuous measurements. ${ }^{9)}$ The Gaussian and mixture Gaussian are continuous probability density functions. Therefore, the Gaussian or mixture Gaussian approximation of the measurement noise of SBIRC is obviously unreasonable and may lead to poor tracking performance. In order to improve the accuracy of trajectory estimation of a target in the boost stage, a more appropriate description of the SBIRC measurement noise and relevant estimation methods are therefore necessary to be considered.

Quantization is a word in digital signal processing (DSP). It is generally recognized that it is caused by finite computation precision in microprocessors or the limit of bandwidth. $^{10,11)}$ The statistical theory of quantization was well studied by Widrow et al.: ${ }^{12)}$ the definition of the quantizer was given and the statistical analysis of the quantization noise was carried out. Estimation with quantized measurements has been studied for decades. As shown in Asmar et al., ${ }^{13)}$ the authors compared several filters for vertical state estimation with quantized barometric altitude and illustrate the poor performance of conventional Kalman filters in the presence of quantization noise. Karlsson et al. treated the quantization noise as an additional uniform noise based on the additive uniform noise (AUN) assumption, ${ }^{14)}$ therefore increasing the measurement covariance in the filtering. Curry et al. presented an approach for quantized measurement by computing moments conditioned on quantized measurement, ${ }^{11)}$ which requires complex quadrature for implementation. To compute the conditional mean and covariance efficiently, Duan et al. proposed a numerical algorithm for approximating MMSE estimation using quantized measurement, ${ }^{15)}$ in which the gridding method is utilized to calculate the integrals. However, in the multidimensional case, the gridding method uses Cartesian products of one-dimensional integration rules, which suffers from the curse of dimensionality.

Among various estimation algorithms, the extended Kalman filtering (EKF), which is based on first-order linearization of the non-linear state model and measurement model, is widely used. ${ }^{16)}$ Many studies have applied the cubature Kalman filter to target tracking problems ${ }^{17-19)}$ in which the spherical-radial cubature rule is used to compute statistics of a nonlinearly transformed Gaussian random variable.
Some studies applied particle filtering, but with heavy computational burden. ${ }^{20-22)}$

In this paper, the characteristics of SBIRC measurements are analyzed theoretically. Using the mid-riser quantizer in digital signal processing to describe the discrete image plane, the measurement noise model of a SBIRC is given. To deal with the quantized measurements of SBIRCs, quantized extended Kalman filtering (QEKF) is proposed, in which the measurement update is derived based on the conditional mean estimate given the quantized measurements. To avoid the curse of dimensionality when calculating the integrals in measurement updates, the Genz's transformation method is used to transform the original integral into an integral over a unit hyper-cube. ${ }^{23,24)}$ The quasi-Monte Carlo (QMC) method is then used to efficiently calculate the integrals. To further improve the tracking accuracy, a quantized high-degree cubature Kalman filtering (QHCKF) is developed by integrating the fifth-degree cubature rule into the framework of the QEKF.

The main contributions of this paper are: 1) Development of the SBIRC measurement model and analysis of its measurement noise (i.e., we first propose to use the mid-riser quantizer to model the discrete image plane of SBIRC); 2) state estimation methods with quantized measurements for target tracking; and 3) a combination of the EKF/highdegree cubature rule and the QMC integration method to avoid the curse of dimensionality.

The paper is outlined as follows. Section 2 introduces the SBIRC-based target tracking problem and develops the dynamic model and measurement model of SBIRCs. Section 3 introduces the proposed QEKF and QHCKF algorithms. The simulation conditions and results are shown in Section 4. Finally, the conclusions are drawn in Section 5.

\section{Problem Statement}

In this section, the SBIRC-based target tracking problem is described. Section 2.1 gives the dynamic model of a target in the boost phase. In Section 2.2, the derivation of SBIRCbased measurement equations is given. Different from most existing works, it is shown that the SBIRC-based measurements are corrupted by not only Gaussian noise, but also quantization noise. To describe the non-Gaussian measurement noise, a quantizer-based SBIRC measurement model is obtained by analyzing the working principles of SBIRCs.

\subsection{Dynamic model of target in the boost phase}

Target acceleration during the boost phase can be expressed as

$$
\boldsymbol{a}=\boldsymbol{a}_{N}+\boldsymbol{a}_{G}
$$

where, $\boldsymbol{a}_{N}$ denotes the net acceleration of the thrust $T$ and drag $D$ and $\boldsymbol{a}_{G}$ denotes the acceleration of gravity.

In this paper, the gravity-turn model is presented to describe the boost phase motion of the target, ${ }^{8)}$ in which the thrust and drag are assumed to be parallel to the relative velocity vector $\boldsymbol{v}_{r}$ in an Earth-center/Earth-fixed coordinate system (ECEF-CS). ${ }^{25}$ ) Thus, we have 


$$
\boldsymbol{a}_{N}=\left(\alpha / v_{r}\right) \boldsymbol{v}_{r}
$$

where, $\alpha=(T-D) / m$ denotes the magnitude of the net acceleration of the thrust and drag, $m$ denotes the target mass, and $v_{r}=\left\|\boldsymbol{v}_{r}\right\|$ is the target speed.

Let $[x, y, z]$ denote the coordinate of the target in ECI-CS. We have

$$
[\ddot{x}, \ddot{y}, \ddot{z}]^{T}=\frac{\alpha}{v_{r}}\left[\dot{x}-\omega_{e} y, \dot{y}+\omega_{e} x, \dot{z}\right]^{T}
$$

where, $\omega_{e}$ is the Earth rotation rate and

$$
v_{r}=\sqrt{\left(\dot{x}-\omega_{e} y\right)^{2}+\left(\dot{y}+\omega_{e} x\right)^{2}+(\dot{z})^{2}}
$$

Note that $\alpha$ is unknown and needs to be estimated. To derive the dynamic model of $\alpha$, we make the following assumptions:

1. The magnitude of the non-gravitational net forces $F(t) \triangleq T(t)-D(t)=F\left(t_{0}\right)$ is constant; where, $t_{0}$ is an arbitrary initial time.

2. The target mass $m$ is linearly decreasing at a constant rate (i.e., $\dot{m}=-\delta_{m}$ ); where, $\delta_{m}$ is a constant value.

We have, for $t \geq t_{0}$

$$
\begin{aligned}
\alpha(t) & =\frac{F(t)}{m(t)} \\
& =\frac{F\left(t_{0}\right)}{m\left(t_{0}\right)-\delta_{m}\left(t-t_{0}\right)} \\
& =\frac{\alpha\left(t_{0}\right)}{1-\beta\left(t_{0}\right)\left(t-t_{0}\right)} \\
\beta(t) & \triangleq \frac{\delta_{m}}{m(t)} \\
& =\frac{\delta_{m}}{m\left(t_{0}\right)-\delta_{m}\left(t-t_{0}\right)} \\
& =\frac{\beta\left(t_{0}\right)}{1-\beta\left(t_{0}\right)\left(t-t_{0}\right)}
\end{aligned}
$$

where, $\beta(t)$ is an auxiliary variable, and it indicates the proportion of the mass reduced from the total mass of the target.

Calculating the derivatives of Eq. (5) and Eq. (6), we get

$$
\begin{gathered}
\dot{\alpha}=\alpha \cdot \beta \\
\dot{\beta}=\beta^{2}
\end{gathered}
$$

According to the well-known inverse-square model of gravity, the acceleration of gravity can be expressed as $\boldsymbol{a}_{G}=-\left(\mu / r^{3}\right) \boldsymbol{r}$; where, $\mu$ is the gravitational parameter, $\boldsymbol{r}=\left[\begin{array}{lll}x & y & z\end{array}\right]^{T}$ and $r=\|\boldsymbol{r}\|$. Then, the gravity-turn dynamic model in the Earth-center inertial coordinate system (ECI$\mathrm{CS})$ is given as follows:

$$
\begin{aligned}
& {[\dot{x}, \dot{y}, \dot{z}]^{T}=\left[\begin{array}{lll}
v_{x} & v_{y} & v_{z}
\end{array}\right]^{T}} \\
& {[\ddot{x}, \ddot{y}, \ddot{z}]^{T}=\frac{\alpha}{v_{r}}\left[\dot{x}-\omega_{e} y, \dot{y}+\omega_{e} x, \dot{z}\right]^{T}} \\
& -\left(\mu / r^{3}\right)[x, y, z]^{T}
\end{aligned}
$$

$$
\begin{gathered}
\dot{\alpha}=\alpha \cdot \beta \\
\dot{\beta}=\beta^{2}
\end{gathered}
$$

The state vector $x$ is defined as

$$
\boldsymbol{x}=\left[x, y, z, v_{x}, v_{y}, v_{z}, \alpha, \beta\right]^{T}
$$

According to Eqs. (9), (10), (11) and (12), the dynamic model is as follows:

$$
\dot{\boldsymbol{x}}=\left[\begin{array}{c}
v_{x} \\
v_{y} \\
v_{z} \\
\frac{\alpha}{v_{r}}\left(\dot{x}-\omega_{e} y\right)-\frac{\mu}{r^{3}} x \\
\frac{\alpha}{v_{r}}\left(\dot{y}-\omega_{e} x\right)-\frac{\mu}{r^{3}} y \\
\frac{\alpha}{v_{r}} \dot{z}-\frac{\mu}{r^{3}} z \\
\alpha \cdot \beta \\
\beta^{2}
\end{array}\right]
$$

\subsection{SBIRC-based measurements}

SBIRCs are commonly used for target tracking in the boost stage and use computer vision and a photogrammetry technique to determine the location of the target. In real applications, at least two satellites are used simultaneously for target tracking, and each satellite is equipped with one SBIRC (see Fig. 1). To represent the statistical properties of the SBIRC noise measured, Gaussian distribution is widely used. ${ }^{6,7)}$ However, the image plane of the SBIRC is composed of discrete pixels, which leads to quantized measurements; that is, the image point is not consistent on the image plane. In order to improve target tracking accuracy, the characteristics of SBIRC measurement need to be theoretically analyzed rather than assumed as Gaussian distribution, which will be given below.

\subsubsection{Derivation of measurement equations}

To derive the measurement equations, we make the following assumptions:

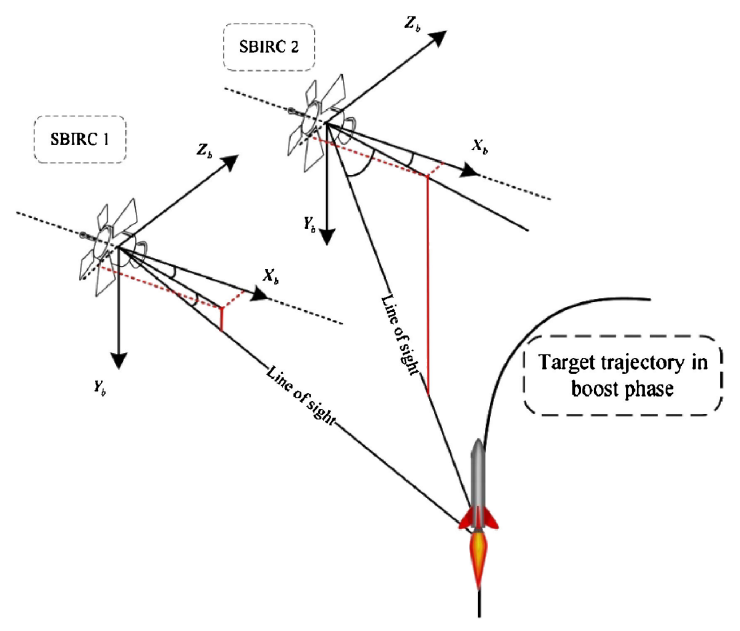

Fig. 1. Target tracking using SBIRCs. 


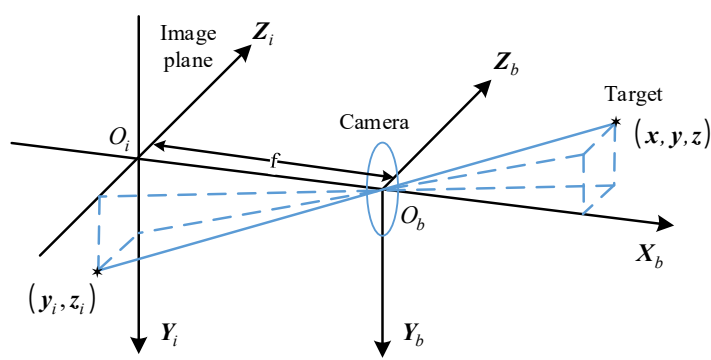

Fig. 2. Geometry of image plane from the infrared camera.

1) The camera coordinate system coincides with the body coordinate system of satellite $O_{b} \boldsymbol{X}_{b} \boldsymbol{Y}_{b} \boldsymbol{Z}_{b}$.

2) The SBIRCs are simple pinhole cameras, which means the target space point $(x, y, z)$, camera perspective center $O_{b}$, and image point $\left(y_{i}, z_{i}\right)$ lie in a straight line (see Fig. 2).

3) The image plane coordinate system $O_{i} \boldsymbol{Y}_{i} \boldsymbol{Z}_{i}$ (in mm) coincides with the pixel coordinate system $O_{p} \boldsymbol{Y}_{p} \boldsymbol{Z}_{p}$ (in pixels).

Let $\boldsymbol{y}_{k}^{i}$ denote the ideal measurement of the $i$ th satellite at time $k$. $\boldsymbol{y}_{k}^{i}$ consists of $\boldsymbol{y}_{p}^{i}$ (i.e., pixel coordinate of the image point in the $y$ direction) and $z_{p}^{i}$ (i.e., pixel coordinate of image point in the $z$ direction). According to the transformation between the body coordinate system and the pixel coordinate system, we get

$$
\left[\begin{array}{c}
y_{p}^{i} \\
z_{p}^{i}
\end{array}\right]=\left[\begin{array}{c}
-f \frac{y_{b}^{i}}{\varepsilon_{y} \cdot x_{b}^{i}} \\
-f \frac{z_{b}^{i}}{\varepsilon_{z} \cdot x_{b}^{i}}
\end{array}\right]
$$

where, $\left(x_{b}^{i}, y_{b}^{i}, z_{b}^{i}\right)$ is the position of the target in the body coordinates of the satellite $i, f$ is the focal length of SBIRC, $\varepsilon_{y}$ and $\varepsilon_{z}$ are the length of the pixel in the direction of $\boldsymbol{y}_{p}$ and $z_{p}$, respectively.

Then, according to the transformation between the body coordinate system and ECEF-CS, we get

$$
\left[\begin{array}{c}
x_{b} \\
y_{b} \\
z_{b}
\end{array}\right]=C_{e}^{b}\left[\begin{array}{c}
x-x_{s} \\
y-y_{s} \\
z-z_{s}
\end{array}\right]
$$

where, $C_{e}^{b}$ is the transformation matrix from the ECEF-CS to the body coordinate system, $(x, y, z)$ is the position of the target in the ECEF-CS, and $\left(x_{s}, y_{s}, z_{s}\right)$ is the position of the satellite in the ECEF-CS.

Thus, the ideal measurement $\boldsymbol{y}_{k}^{i}$ is given as

$$
\begin{aligned}
\boldsymbol{y}_{k}^{i}= & {\left[\begin{array}{c}
y_{p}^{i} \\
z_{p}^{i}
\end{array}\right] } \\
= & {\left[\begin{array}{c}
-\frac{f}{\varepsilon_{y}} \cdot \frac{C_{21}^{i}\left(x-x_{s}^{i}\right)+C_{22}^{i}\left(y-y_{s}^{i}\right)+C_{23}^{i}\left(z-z_{s}^{i}\right)}{C_{11}^{i}\left(x-x_{s}^{i}\right)+C_{12}^{i}\left(y-y_{s}^{i}\right)+C_{13}^{i}\left(z-z_{s}^{i}\right)} \\
-\frac{f}{\varepsilon_{z}} \cdot \frac{C_{31}^{i}\left(x-x_{s}^{i}\right)+C_{32}^{i}\left(y-y_{s}^{i}\right)+C_{33}^{i}\left(z-z_{s}^{i}\right)}{C_{11}^{i}\left(x-x_{s}^{i}\right)+C_{12}^{i}\left(y-y_{s}^{i}\right)+C_{13}^{i}\left(z-z_{s}^{i}\right)}
\end{array}\right] }
\end{aligned}
$$

where, the superscript $i$ denotes the $i$ th satellite, and $C_{a b}^{i}$ denotes the value at the $a$ th row and the $b$ th column of the transformation matrix $C_{e}^{b}$.

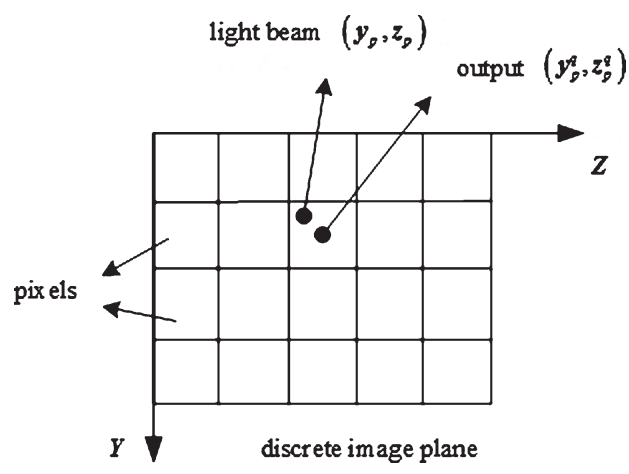

Fig. 3. The discrete image plane of the SBIRCs.

\subsubsection{SBIRC noise measurement model}

For the discrete image plane of SBIRC, a light beam illuminating within a pixel will yield a coordinate output corresponding to the center of that pixel (see Fig. 3). ${ }^{26,27)}$ Therefore, the coordinates of the target in the pixel coordinate system $\left(y_{p}^{q}, z_{p}^{q}\right)$ can suffer from quantization errors of up to 0.5 pixels (i.e., $-0.5 \leq y_{p}^{q}-y_{p}<0.5$ and $-0.5 \leq z_{p}^{q}-$ $z_{p}<0.5$; where, $\left(y_{p}, z_{p}\right)$ is the ideal coordinate of the target in the pixel coordinate system).

From the output of the discrete image plane, the only thing we can determine is the pixel in which the actual coordinate $\left(y_{p}, z_{p}\right)$ is located. It is not possible to specify the actual coordinate any more precisely than containment within the pixel. Therefore, the SBIRC measurements are "imprecise measurements" and are highly non-Gaussian. (Details of "imprecise measurements" can be seen in Karlsson and Gustafsson.) $)^{27)}$

Based on the above analysis, a mid-riser quantizer is used to model the discrete image plane of the SBIRC. The coordinate output of the SBIRC can be seen as the output of a midriser quantizer.

In digital signal processing, a quantizer is defined as a nonlinear operator having the input-output staircase relation. (Details of the quantizer can be seen in Widrow and István. ${ }^{28)}$ The mathematical expression of the mid-riser quantizer is as follows

$$
\boldsymbol{y}^{q}=Q(\boldsymbol{y})=\Delta\left\lfloor\frac{\boldsymbol{y}}{\Delta}\right\rfloor+\frac{\Delta}{2}
$$

where, $\boldsymbol{Q}$ denotes the mid-riser quantizer, $\boldsymbol{y}$ denotes the input of the quantizer, the $\lfloor\cdot\rfloor$ operator rounds downward to the nearest integer, and $\Delta$ denotes the length of the quantization interval. From the output of the quantizer $\boldsymbol{y}^{q}$, the only thing we can infer is that $\boldsymbol{y} \in A_{i}$; where, $A_{i}=\left[\boldsymbol{a}_{k}, \boldsymbol{b}_{k}\right)$ is the $i$ th quantization interval, which is known. The characteristics of the mid-riser quantizer can be seen in Fig. 4.

Based on the mid-riser quantizer, the relationship between the ideal coordinates and quantized coordinates of the target in the pixel coordinate system can be expressed as

$$
\begin{aligned}
& y_{p}^{q}=\mathbf{Q}\left(\tilde{y}_{p}\right)=\mathbf{Q}\left(y_{p}+w_{y}\right)=\tilde{y}_{p}+e_{y} \\
& z_{p}^{q}=\mathbf{Q}\left(\tilde{z}_{p}\right)=\mathbf{Q}\left(z_{p}+w_{z}\right)=\tilde{z}_{p}+e_{z}
\end{aligned}
$$

where, $w_{y}$ and $w_{z}$ are zero-mean Gaussian white noise caused 


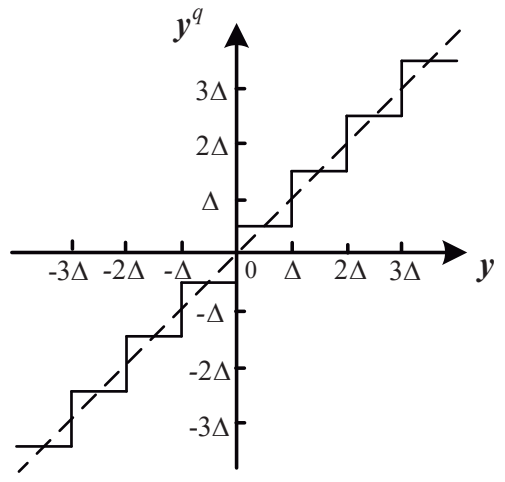

Fig. 4. The mid-riser quantizer (1-dimensional case).

by parameters such as poor illumination and high temperature, $e_{y}$ and $e_{z}$ are the quantization noise, which depends on the size of pixels, $y_{p}^{q}$ and $z_{p}^{q}$ are measurements after quantization, which can be obtained directly from the sensor, $\tilde{y}_{p}$ and $\tilde{z}_{p}$ are the measurements before quantization, and $y_{p}$ and $z_{p}$ are the real measurements.

It can be seen from Eq. (19) that the SBIRC noise measurement consists of two parts: 1) Gaussian noise $w_{y}$ and $w_{z}$, and 2) quantization noise $e_{y}$ and $e_{z}$. For the case that the Gaussian noise is larger (i.e., quantization noise has less influence on measurements), traditional EKF is adoptable. However, when the quantization noise is larger, the nonGaussian property of the measurements makes traditional EKF unadoptable, which motivates our work.

Substituting Eq. (17) into Eq. (19), we get the measurement equation for satellite $i$

$$
\begin{aligned}
\boldsymbol{y}_{k}^{q}= & {\left[\begin{array}{c}
y_{p}^{q} \\
z_{p}^{q}
\end{array}\right] } \\
= & {\left[\begin{array}{c}
-\frac{f}{\varepsilon_{y}} \cdot \frac{C_{21}^{i} \Delta x^{i}+C_{22}^{i} \Delta y^{i}+C_{23}^{i} \Delta z^{i}}{C_{11}^{i} \Delta x^{i}+C_{12}^{i} \Delta y^{i}+C_{13}^{i} \Delta z^{i}}+w_{y}^{i} \\
-\frac{f}{\varepsilon_{z}} \cdot \frac{C_{31}^{i} \Delta x^{i}+C_{32}^{i} \Delta y^{i}+C_{33}^{i} \Delta z^{i}}{C_{11}^{i} \Delta x^{i}+C_{12}^{i} \Delta y^{i}+C_{13}^{i} \Delta z^{i}}+w_{z}^{i}
\end{array}\right] } \\
& +\left[\begin{array}{c}
e_{y}^{i} \\
e_{z}^{i}
\end{array}\right]
\end{aligned}
$$

where, $\Delta x^{i}=x-x_{s}^{i}, \Delta y^{i}=y-y_{s}^{i}, \Delta z^{i}=z-z_{s}^{i}, w_{y}^{i}$ and $w_{z}^{i}$ are the Gaussian part measurement noise of satellite $i$, and $e_{y}^{i}$ and $e_{z}^{i}$ are the quantization part. Therefore, the measurement noise is non-Gaussian. The focus of this paper is on the state estimation methods dealing with this kind of non-Gaussian noise for target tracking using SBIRCs.

\section{Filtering with Quantized Measurements}

Consider the following nonlinear discrete system model with quantized measurements

$$
\begin{gathered}
\boldsymbol{x}_{k+1}=\boldsymbol{f}\left(\boldsymbol{x}_{k}\right)+\boldsymbol{v}_{k} \\
\boldsymbol{y}_{k}=\boldsymbol{h}\left(\boldsymbol{x}_{k}\right)+\boldsymbol{w}_{k} \\
\boldsymbol{y}_{k}^{q}=\boldsymbol{Q}\left(\boldsymbol{y}_{k}\right)=\boldsymbol{y}_{k}+\boldsymbol{e}_{k}
\end{gathered}
$$

where, $\boldsymbol{x}_{k} \in \mathbf{R}^{n}$ is the state vector of the dynamic system, $\boldsymbol{y}_{k} \in \mathbf{R}^{m}$ is the measurement vector before quantization, which is unknown, $\boldsymbol{v}_{k}$ and $\boldsymbol{w}_{k}$ are mutually independent zero-mean Gaussian noise with covariance $\boldsymbol{Q}_{k}$ and $\boldsymbol{R}_{k}$, subscript $k$ represents discrete time $t_{k}, \boldsymbol{f}$ and $\boldsymbol{h}$ are the nonlinear state function and measurement function of the system, respectively, symbol $\boldsymbol{Q}$ denotes the mid-riser quantization operator, $\boldsymbol{y}_{k}^{q}$ is the measurement vector after quantization which is what can be obtained directly from the sensor, and $\boldsymbol{e}_{k}$ is the quantization noise.

Note that, even if measurement function $\boldsymbol{h}$ is linear and $\boldsymbol{w}_{k}$ is Gaussian, $\boldsymbol{Q}\left(\boldsymbol{y}_{k}\right)$ will be nonlinear and $\boldsymbol{e}_{k}$ will be nonGaussian because of the quantization operator.

\subsection{Review of extended Kalman filtering}

It is well known that Kalman filtering is capable of achieving optimal solutions for estimation problems of linear systems with Gaussian noise. Furthermore, EKF is a generalization of $\mathrm{KF}$ for nonlinear systems. ${ }^{30)}$ Considering the nonlinear discrete system model shown in Eq. (21) and Eq. (22), the EKF is given by the following equations.

Define

$$
\boldsymbol{Y}^{k} \triangleq\{\boldsymbol{y}, i \leq k\}
$$

EKF time update

Given the latest state $\hat{\boldsymbol{x}}_{k-1 \mid k-1}$, the predicted state $\hat{\boldsymbol{x}}_{k \mid k-1}$ is

$$
\hat{\boldsymbol{x}}_{k \mid k-1}=\boldsymbol{f}\left(\hat{\boldsymbol{x}}_{k-1 \mid k-1}\right)
$$

Similarly, the predicted measurement $\hat{\boldsymbol{y}}_{k}$ is

$$
\hat{\boldsymbol{y}}_{k}=\boldsymbol{h}\left(\hat{\boldsymbol{x}}_{k \mid k-1}\right)
$$

The state prediction covariance $\boldsymbol{P}_{k \mid k-1}$ is

$$
\boldsymbol{P}_{k \mid k-1}=\boldsymbol{F}_{k-1} \boldsymbol{P}_{k-1 \mid k-1} \boldsymbol{F}_{k-1}^{T}+\boldsymbol{Q}_{k-1}
$$

where, $\boldsymbol{F}_{k-1}$ is the Jacobian of the vector $\boldsymbol{f}$ evaluated at the latest estimate of the state, and $\boldsymbol{P}_{k-1 \mid k-1}$ is covariance matrix at time $k-1$.

EKF measurement update

The innovation covariance $S_{k}$ is

$$
\boldsymbol{S}_{k}=\boldsymbol{R}_{k}+\boldsymbol{H}_{k} \boldsymbol{P}_{k \mid k-1} \boldsymbol{H}_{k}^{T}
$$

where, $\boldsymbol{H}_{k}$ is the Jacobian of the measurement function $\boldsymbol{h}$.

The filter gain $\boldsymbol{K}_{k}$ is

$$
\boldsymbol{K}_{k}=\boldsymbol{P}_{k \mid k-1} \boldsymbol{H}_{k}^{T} \boldsymbol{S}_{k}^{-1}
$$

The update equation for state and its covariance are

$$
\begin{aligned}
\hat{\boldsymbol{x}}_{k \mid k} & =\boldsymbol{E}\left(\boldsymbol{x}_{k} \mid \boldsymbol{Y}^{k}\right) \\
= & \hat{\boldsymbol{x}}_{k \mid k-1}+\boldsymbol{K}_{k}\left(\boldsymbol{y}_{k}-\hat{\boldsymbol{y}}_{k}\right) \\
\boldsymbol{P}_{k \mid k} & =\operatorname{MSE}\left(\hat{\boldsymbol{x}}_{k \mid k} \mid \boldsymbol{Y}^{k}\right) \\
& =\boldsymbol{P}_{k \mid k-1}-\boldsymbol{K}_{k} \boldsymbol{S}_{k} \boldsymbol{K}_{k}^{T}
\end{aligned}
$$

\subsection{Quantized extended Kalman filtering}

Consider the nonlinear discrete system model with quantized measurements shown in Eqs. (21)-(23), the EKF suffers from poor performance due to the mismatching Gauss hypothesis. In this section, an improved algorithm named 
QEKF is developed to deal with the quantized measurements. Given $\hat{\boldsymbol{x}}_{k-1 \mid k-1}$ and $\boldsymbol{P}_{k-1 \mid k-1}$, under the Gaussian assumption of a prior probability density function (pdf), the time update equations of QEKF are the same as that of EKF (i.e., Eqs. (25)-(27)) because of the Markov property of the system. The differences between the QEKF algorithm proposed and the conventional EKF algorithm lie in the measurement update, which will be introduced below.

\subsubsection{Measurement update}

Define the sequence of quantized measurements $\boldsymbol{Y}_{k}^{q} \triangleq$ $\left\{\boldsymbol{y}_{i}^{q}, i \leq k\right\}$. Using the minimum square error estimation theory and property of conditional expectation, the state estimation $\hat{\boldsymbol{x}}_{k \mid k}$ based on the quantized measurement $\boldsymbol{Y}_{k}^{q}$ is calculated as follows ${ }^{15)}$

$$
\begin{aligned}
\hat{\boldsymbol{x}}_{k \mid k} & =E\left[\boldsymbol{x}_{k} \mid \boldsymbol{Y}_{k}^{q}\right] \\
& =E\left[\boldsymbol{x}_{k} \mid \boldsymbol{Y}_{k-1}^{q}, \boldsymbol{y}_{k}^{q}\right] \\
& =E\left[\boldsymbol{x}_{k} \mid \boldsymbol{Y}_{k-1}^{q}, \boldsymbol{y}_{k} \in A_{i}\right] \\
& =E\left[E\left(\boldsymbol{x}_{k} \mid \boldsymbol{Y}_{k-1}^{q}, \boldsymbol{y}_{k}\right) \mid \boldsymbol{Y}_{k-1}^{q}, \boldsymbol{y}_{k} \in A_{i}\right]
\end{aligned}
$$

where, $E\left(\boldsymbol{x}_{k} \mid \boldsymbol{Y}_{k-1}^{q}, \boldsymbol{y}_{k}\right)$ is the conditional expectation of $\boldsymbol{x}_{k}$ given former quantized measurements $\boldsymbol{Y}_{k-1}^{q}$ and current measurement $\boldsymbol{y}_{k}$, which can be obtained using Eq. (30). Therefore,

$$
\hat{\boldsymbol{x}}_{k \mid k}=\hat{\boldsymbol{x}}_{k \mid k-1}+K_{k}\left[E\left[\boldsymbol{y}_{k} \mid \boldsymbol{Y}_{k-1}^{q}, \boldsymbol{y}_{k} \in A_{i}\right]-\hat{\boldsymbol{y}}_{k}\right]
$$

where,

$$
\begin{aligned}
E[ & {\left[\boldsymbol{y}_{k} \mid \boldsymbol{Y}_{k-1}^{q}, \boldsymbol{y}_{k} \in A_{i}\right] } \\
& =\int_{\boldsymbol{a}_{k}}^{\boldsymbol{b}_{k}} \boldsymbol{y}_{k} p\left(\boldsymbol{y}_{k} \mid \boldsymbol{Y}_{k-1}^{q}, \boldsymbol{y}_{k} \in A_{i}\right) d \boldsymbol{y}_{k}
\end{aligned}
$$

and $K_{k}$ can be calculated using Eq. (29).

Furthermore, the covariance $\boldsymbol{P}_{k \mid k}$ is ${ }^{15)}$

$$
\begin{aligned}
\boldsymbol{P}_{k \mid k} & =\operatorname{MSE}\left(\boldsymbol{x}_{k} \mid \boldsymbol{Y}_{k-1}^{q}, \boldsymbol{y}_{k}^{q}\right) \\
& =\operatorname{MSE}\left(\boldsymbol{x}_{k} \mid \boldsymbol{Y}_{k-1}^{q}, \boldsymbol{y}_{k} \in A_{i}\right) \\
& =E\left[\left(\boldsymbol{x}_{k}-\hat{\boldsymbol{x}}_{k \mid k}\right)\left(\boldsymbol{x}_{k}-\hat{\boldsymbol{x}}_{k \mid k}\right)^{T} \mid \boldsymbol{Y}_{k-1}^{q}, \boldsymbol{y}_{k} \in A_{i}\right]
\end{aligned}
$$

where,

$$
\begin{aligned}
\boldsymbol{x}_{k}-\hat{\boldsymbol{x}}_{k \mid k}= & \boldsymbol{x}_{k}-E\left[\boldsymbol{x}_{k} \mid \boldsymbol{Y}_{k-1}^{q}, \boldsymbol{y}_{k}\right] \\
& +E\left[\boldsymbol{x}_{k} \mid \boldsymbol{Y}_{k-1}^{q}, \boldsymbol{y}_{k}\right]-\hat{\boldsymbol{x}}_{k \mid k} \\
= & \boldsymbol{x}_{k}-E\left[\boldsymbol{x}_{k} \mid \boldsymbol{Y}_{k-1}^{q}, \boldsymbol{y}_{k}\right] \\
& +\left(\hat{\boldsymbol{x}}_{k \mid k-1}+K_{k}\left[\boldsymbol{y}_{k}-\hat{\boldsymbol{y}}_{k}\right]\right)-\hat{\boldsymbol{x}}_{k \mid k} \\
= & \boldsymbol{x}_{k}-E\left[\boldsymbol{x}_{k} \mid \boldsymbol{Y}_{k-1}^{q}, \boldsymbol{y}_{k}\right] \\
& +K_{k}\left(\boldsymbol{y}_{k}-E\left[\boldsymbol{y}_{k} \mid \boldsymbol{Y}_{k-1}^{q}, \boldsymbol{y}_{k} \in A_{i}\right]\right)
\end{aligned}
$$

Then,

$$
\begin{aligned}
\boldsymbol{P}_{k \mid k}= & M S E\left(\boldsymbol{x}_{k} \mid \boldsymbol{Y}_{k-1}^{q}, \boldsymbol{y}_{k}\right) \\
& +K_{k}\left(\boldsymbol{y}_{k}-E\left[\boldsymbol{y}_{k} \mid \boldsymbol{Y}_{k-1}^{q}, \boldsymbol{y}_{k} \in A_{i}\right]\right) \\
& \cdot\left(\boldsymbol{y}_{k}-E\left[\boldsymbol{y}_{k} \mid \boldsymbol{Y}_{k-1}^{q}, \boldsymbol{y}_{k} \in A_{i}\right]\right)^{T} K_{k}^{T} \\
= & M S E\left(\boldsymbol{x}_{k} \mid \boldsymbol{Y}_{k-1}^{q}, \boldsymbol{y}_{k}\right) \\
& +K_{k} \operatorname{cov}\left(\boldsymbol{y}_{k} \mid \boldsymbol{Y}_{k-1}^{q}, \boldsymbol{y}_{k} \in A_{i}\right) K_{k}^{T}
\end{aligned}
$$

where, $\operatorname{MSE}\left(\boldsymbol{x}_{k} \mid \boldsymbol{Y}_{k-1}^{q}, \boldsymbol{y}_{k}\right)$ can be calculated using Eq. (31); that is,

$$
\operatorname{MSE}\left(\boldsymbol{x}_{k} \mid \boldsymbol{Y}_{k-1}^{q}, \boldsymbol{y}_{k}\right)=P_{k \mid k-1}-K_{k} \boldsymbol{S}_{k} K_{k}^{T}
$$

and

$$
\begin{aligned}
\operatorname{cov}( & \left.\boldsymbol{y}_{k} \mid \boldsymbol{Y}_{k-1}^{q}, \boldsymbol{y}_{k} \in A_{i}\right) \\
= & E\left[\boldsymbol{y}_{k} \boldsymbol{y}_{k}^{T} \mid \boldsymbol{Y}_{k-1}^{q}, \boldsymbol{y}_{k} \in A_{i}\right] \\
& -E\left[\boldsymbol{y}_{k} \mid \boldsymbol{Y}_{k-1}^{q}, \boldsymbol{y}_{k} \in A_{i}\right] \cdot E^{T}\left[\boldsymbol{y}_{k} \mid \boldsymbol{Y}_{k-1}^{q}, \boldsymbol{y}_{k} \in A_{i}\right] \\
= & \int_{\boldsymbol{a}_{k}}^{\boldsymbol{b}_{k}} \boldsymbol{y}_{k} \boldsymbol{y}_{k}^{T} p\left(\boldsymbol{y}_{k} \mid \boldsymbol{Y}_{k-1}^{q}, \boldsymbol{y}_{k} \in A_{i}\right) d \boldsymbol{y}_{k} \\
& -E\left[\boldsymbol{y}_{k} \mid \boldsymbol{Y}_{k-1}^{q}, \boldsymbol{y}_{k} \in A_{i}\right] \cdot E^{T}\left[\boldsymbol{y}_{k} \mid \boldsymbol{Y}_{k-1}^{q}, \boldsymbol{y}_{k} \in A_{i}\right]
\end{aligned}
$$

Therefore, the measurement update is given using Eq. (28), Eq. (29), Eq. (37) and Eq. (38). However, we need to find a way to compute the integrals in Eq. (34) and Eq. (39) ; that is,

$$
\int_{\boldsymbol{a}_{k}}^{\boldsymbol{b}_{k}} \boldsymbol{y}_{k} p\left(\boldsymbol{y}_{k} \mid \boldsymbol{Y}_{k-1}^{q}, \boldsymbol{y}_{k} \in A_{i}\right) d \boldsymbol{y}_{k}
$$

and

$$
\int_{\boldsymbol{a}_{k}}^{\boldsymbol{b}_{k}} \boldsymbol{y}_{k} \boldsymbol{y}_{k}^{T} p\left(\boldsymbol{y}_{k} \mid \boldsymbol{Y}_{k-1}^{q}, \boldsymbol{y}_{k} \in A_{i}\right) d \boldsymbol{y}_{k}
$$

It can be seen that both of the integrals include $p\left(\boldsymbol{y}_{k} \mid \boldsymbol{Y}_{k-1}^{q}, \boldsymbol{y}_{k} \in A_{i}\right)$, which is the pdf for $\boldsymbol{y}_{k}$ conditioned on measurements $\boldsymbol{Y}_{k-1}^{q}$ and $\boldsymbol{y}_{k} \in A_{i}$. The prior probability density of the measurement vector is calculated using

$$
\begin{aligned}
p\left(\boldsymbol{y}_{k} \mid \boldsymbol{Y}_{k-1}^{q}\right) & \\
& =\int p\left(\boldsymbol{y}_{k} \mid \boldsymbol{x}_{k}, \boldsymbol{Y}_{k-1}^{q}\right) p\left(\boldsymbol{x}_{k} \mid \boldsymbol{Y}_{k-1}^{q}\right) d \boldsymbol{x}_{k} \\
& =\int \mathcal{N}\left(\boldsymbol{y}_{k} ; \hat{\boldsymbol{y}}_{k \mid k-1}, \boldsymbol{R}_{k}\right) \mathcal{N}\left(\boldsymbol{x}_{k} ; \hat{\boldsymbol{x}}_{k \mid k-1}, P_{k \mid k-1}\right) d \boldsymbol{x}_{k} \\
& =\mathcal{N}\left(\boldsymbol{y}_{k} ; \hat{\boldsymbol{y}}_{k \mid k-1}, \boldsymbol{S}_{k}\right) \\
& =\frac{e^{-\frac{1}{2}\left(\boldsymbol{y}_{k}-\hat{\boldsymbol{y}}_{k \mid k-1}\right)^{T} \boldsymbol{S}_{k}^{-1}\left(\boldsymbol{y}_{k}-\hat{\boldsymbol{y}}_{k \mid k-1}\right)}}{(2 \pi)^{r / 2}\left|\boldsymbol{S}_{k}\right|^{1 / 2}}
\end{aligned}
$$

where, $r$ is the dimension of $\boldsymbol{y}_{k}$ and

$$
P\left(\boldsymbol{y}_{k} \in A_{i} \mid \boldsymbol{Y}_{k-1}^{q}\right)=\int_{\boldsymbol{a}_{k}}^{\boldsymbol{b}_{k}} p\left(\boldsymbol{y}_{k} \mid \boldsymbol{Y}_{k-1}^{q}\right) d \boldsymbol{y}_{k}
$$

Then,

$$
\begin{aligned}
& p\left(\boldsymbol{y}_{k} \mid \boldsymbol{Y}_{k-1}^{q}, \boldsymbol{y}_{k} \in A_{i}\right) \\
& \quad=\left\{\begin{array}{lll}
\frac{p\left(\boldsymbol{y}_{k} \mid \boldsymbol{Y}_{k-1}^{q}\right)}{P\left(\boldsymbol{y}_{k} \in A_{i} \mid \boldsymbol{Y}_{k-1}^{q}\right)} & \text { if } & \boldsymbol{y}_{k} \in A_{i} \\
0 & \text { if } & \boldsymbol{y}_{k} \notin A_{i}
\end{array}\right.
\end{aligned}
$$

Inserting Eqs. (42) and (43) into Eq. (44), we get the expression of $p\left(\boldsymbol{y}_{k} \mid \boldsymbol{Y}_{k-1}^{q}, \boldsymbol{y}_{k} \in A_{i}\right)$.

It is well known that there is no analytical solution to the integrals of Gaussian density in Eqs. (40), (41) and (43). Therefore, a multi-dimensional numerical integration method is needed. This is presented in the next section. 


\subsubsection{Multi-dimensional numerical integration using Genz's transformation and quasi-Monte Carlo method}

The multi-dimensional numerical integration methods can be divided into two categories: product rules and non-product rules. ${ }^{31)}$ Product rules such as the product Gauss-Hermite quadrature rule successively apply the Gauss-Hermite quadrature rule in a tensor-product of one-dimensional integrals. Hence, $m^{n}$ points are needed to compute an $n$-dimensional integral. Non-product rules such as Monte Carlo methods choose points directly from the domain of integration, therefore mitigating the curse of dimensionality.

Clearly, the integrals in Eq. (44) will cause heavy computational burden. Duan et al. ${ }^{15)}$ proposed a numerical way to calculate the integrals using a gridding method in which $m^{n}$ points are used to compute an $n$-dimensional integral. Hence, the computational complexity of the gridding method increases exponentially with the dimension, and therefore suffers from the curse of dimensionality.

In the following, we will introduce the calculation of the integral in Eq. (43). The quasi-Monte Carlo method is used to avoid the curse of dimensionality while calculating the integrals. Before that, we adopt Genz's transformation method $^{23,24)}$ to transform the original integral into an integral over a unit hyper-cube.

The integral in Eq. (43) can be written as

$$
\begin{aligned}
P\left(\boldsymbol{y}_{k} \in A_{i} \mid \boldsymbol{Y}_{k-1}^{q}\right) & \\
= & \int_{\boldsymbol{a}_{k}}^{\boldsymbol{b}_{k}} p\left(\boldsymbol{y}_{k} \mid \boldsymbol{Y}_{k-1}^{q}\right) d \boldsymbol{y}_{k} \\
= & \frac{1}{(2 \pi)^{r / 2}\left|\boldsymbol{S}_{k}\right|^{1 / 2}} \\
& \cdot \int_{a_{1}}^{b_{1}} \int_{a_{2}}^{b_{2}} \cdots \int_{a_{r}}^{b_{r}} e^{-\frac{1}{2}\left(\boldsymbol{y}_{k}-\hat{\boldsymbol{y}}_{k \mid k-1}\right)^{T} \boldsymbol{S}_{k}^{-1}\left(\boldsymbol{y}_{k}-\hat{\boldsymbol{y}}_{k \mid k-1}\right)} d \boldsymbol{y}_{k}
\end{aligned}
$$

Defining $\boldsymbol{\theta}_{k}=\boldsymbol{y}_{k}-\hat{\boldsymbol{y}}_{k \mid k-1}$, we get

$$
\begin{aligned}
& P\left(\boldsymbol{y}_{k} \in A_{i} \mid \boldsymbol{Y}_{k-1}^{q}\right) \\
& \quad=\frac{1}{(2 \pi)^{r / 2}\left|\boldsymbol{S}_{k}\right|^{1 / 2}} \int_{a_{1}^{\prime}}^{b_{1}^{\prime}} \int_{a_{2}^{\prime}}^{b_{2}^{\prime}} \cdots \int_{a_{r}^{\prime}}^{b_{r}^{\prime}} e^{-\frac{1}{2} \boldsymbol{\theta}_{k}^{T} \boldsymbol{S}_{k}^{-1} \boldsymbol{\theta}_{k}} d \boldsymbol{\theta}_{k}
\end{aligned}
$$

where,

$$
\begin{aligned}
& a_{i}^{\prime}=a_{i}-\hat{y}_{k \mid k-1, i} \\
& b_{i}^{\prime}=b_{i}-\hat{y}_{k \mid k-1, i} \quad \text { for } \quad i=1,2, \cdots, r
\end{aligned}
$$

Define $\boldsymbol{\theta}_{k}=\left|S_{k}\right|^{1 / 2} z_{k}$ and substitute $\boldsymbol{\theta}_{k}$ with $z_{k}$, we get

$$
\begin{aligned}
& P\left(\boldsymbol{y}_{k} \in A_{i} \mid \boldsymbol{Y}_{k-1}^{q}\right) \\
& \quad=\frac{1}{(2 \pi)^{r / 2}} \int_{a_{1}^{\prime \prime}}^{b_{1}^{\prime \prime}} e^{-\frac{z_{1}^{2}}{2}} \int_{a_{2}^{\prime \prime}}^{b_{2}^{\prime \prime}} e^{-\frac{z_{2}^{2}}{2}} \cdots \int_{a_{r}^{\prime \prime}}^{b_{r}^{\prime \prime}} e^{-\frac{z_{r}^{2}}{2}} d z
\end{aligned}
$$

where,

$$
\begin{aligned}
& a_{i}^{\prime \prime}=\left(a_{i}^{\prime}-\sum_{j=1}^{i-1}\left|\boldsymbol{S}_{k}\right|_{i j}^{1 / 2} z_{j}\right) /\left|\boldsymbol{S}_{k}\right|_{i i}^{1 / 2} \\
& b_{i}^{\prime \prime}=\left(b_{i}^{\prime}-\sum_{j=1}^{i-1}\left|\boldsymbol{S}_{k}\right|_{i j}^{1 / 2} z_{j}\right) /\left|\boldsymbol{S}_{k}\right|_{i i}^{1 / 2} \quad \text { for } \quad i=1,2, \cdots, r
\end{aligned}
$$

Define $u_{i}=\Phi\left(z_{i}\right)=\frac{1}{\sqrt{2 \pi}} \int_{-\infty}^{z_{i}} e^{-\frac{1}{2} t^{2}} d t$, therefore $d u_{i}=\frac{1}{\sqrt{2 \pi}} e^{-\frac{1}{2} z_{i}^{2}} d z_{i}$. Then,

$$
P\left(\boldsymbol{y}_{k} \in A_{i} \mid \boldsymbol{Y}_{k-1}^{q}\right)=\int_{d_{1}}^{e_{1}} \int_{d_{2}}^{e_{2}} \cdots \int_{d_{r}}^{e_{r}} d \boldsymbol{u}
$$

where, $d_{i}=\Phi\left(a_{i}^{\prime \prime}\right)$ and $e_{i}=\Phi\left(b_{i}^{\prime \prime}\right)$ for $i=1,2, \cdots, r$.

Defining $u_{i}=d_{i}+w_{i}\left(e_{i}-d_{i}\right)$, we get

$$
\begin{aligned}
& P\left(\boldsymbol{y}_{k} \in A_{i} \mid \boldsymbol{Y}_{k-1}^{q}\right) \\
& \quad=\int_{0}^{1}\left(e_{1}-d_{1}\right) \int_{0}^{1}\left(e_{2}-d_{2}\right) \cdots \int_{0}^{1}\left(e_{r}-d_{r}\right) d \boldsymbol{w}
\end{aligned}
$$

Note that $d_{i}$ and $e_{i}$ are related to $u_{1}, u_{2}, \cdots, u_{i-1}$, and not related to $u_{i}$. Therefore, $d_{1}$ and $e_{1}$ are not related to $w$, and $d_{i}$ and $e_{i}$ are not related to $w_{i}{ }^{23)}$ Thus, Eq. (51) can be written as

$$
\begin{aligned}
& P\left(\boldsymbol{y}_{k} \in A_{i} \mid \boldsymbol{Y}_{k-1}^{q}\right) \\
& \quad=\left(e_{1}-d_{1}\right) \int_{0}^{1}\left(e_{2}-d_{2}\right) \cdots \int_{0}^{1}\left(e_{r}-d_{r}\right) \int_{0}^{1} d \boldsymbol{w}
\end{aligned}
$$

Therefore, the number of integration variables is reduced by one.

Then, the $N$-points quasi-Monte Carlo method is used to approximate the integral in Eq. (52).

$$
\begin{aligned}
P\left(\boldsymbol{y}_{k} \in A_{i} \mid \boldsymbol{Y}_{k-1}^{q}\right) & =\int_{[0,1]^{r}} f(\boldsymbol{w}) d \boldsymbol{w} \\
& \approx \frac{1}{N} \sum_{i=1}^{N} f\left(\boldsymbol{w}_{i}\right)
\end{aligned}
$$

where, $\boldsymbol{w}_{i}$ is produced using the Halton sequence, ${ }^{29)}$ $f(\boldsymbol{w})=\left(e_{1}-d_{1}\right)\left(e_{2}-d_{2}\right) \cdots\left(e_{r}-d_{r}\right)$.

For the integrals in Eqs. (40) and (41), only minor modifications to the above algorithm are needed. The general numerical integration algorithm for Eqs. (40), (41) and (43) is given in Table 1.

\subsection{Quantized high-degree cubature Kalman filtering}

As used in EKF, for the QEKF algorithm proposed in Section 3.2, a linearization technique is used to approximate the nonlinear model. To further improve the tracking accuracy, we propose to integrate the fifth-degree cubature rule into the framework of the QEKF to get create QHCKF.

Based on the fifth-degree cubature rule, the time update equations (Eqs. (25)-(27)) can be rewritten as

$$
\begin{gathered}
\hat{\boldsymbol{x}}_{k \mid k-1}=\sum_{i=0}^{2 n^{2}} \omega_{i} \boldsymbol{\chi}_{k \mid k-1, i} \\
\hat{\boldsymbol{y}}_{k \mid k-1}=\sum_{i=0}^{2 n^{2}} \omega_{i} \boldsymbol{\Upsilon}_{k \mid k-1, i} \\
\boldsymbol{P}_{k \mid k-1}=\sum_{i=0}^{2 n^{2}} \omega_{i}\left(\boldsymbol{\chi}_{k \mid k-1, i}-\hat{\boldsymbol{x}}_{k \mid k-1}\right)\left(\boldsymbol{\chi}_{k \mid k-1, i}-\hat{\boldsymbol{x}}_{k \mid k-1}\right)^{T}+\boldsymbol{Q}_{k-1}
\end{gathered}
$$


Table 1. The proposed numerical integration algorithm.

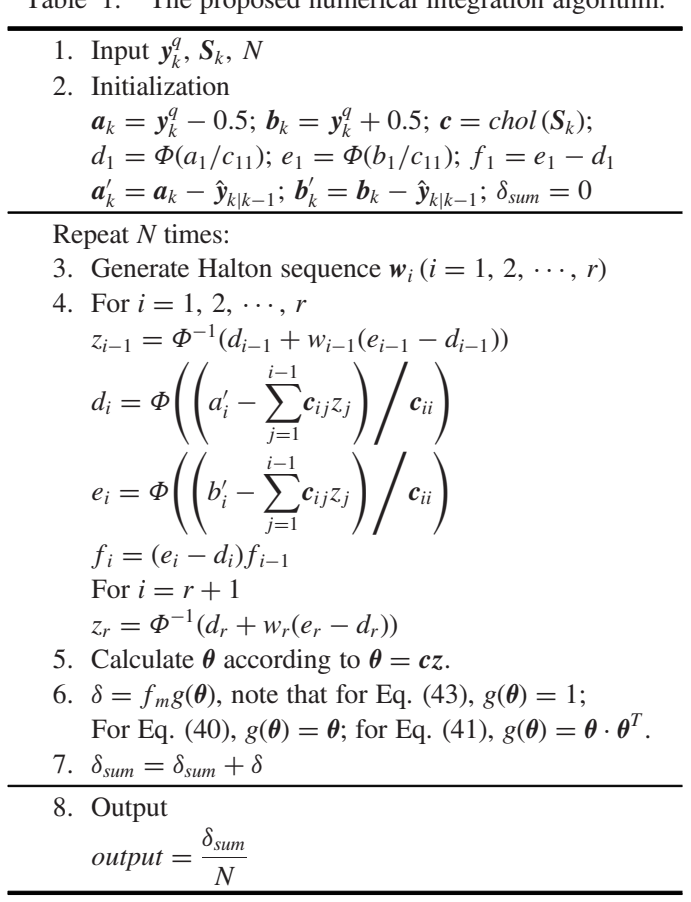

where, $\chi_{k \mid k-1, i}$ are the points transformed from the state model, and $\boldsymbol{\Upsilon}_{k \mid k-1, i}$ are the points transformed from the measurement model:

$$
\begin{gathered}
\chi_{k \mid k-1, i}=\boldsymbol{f}\left(\chi_{k-1 \mid k-1, i}\right), \quad i=0,1, \cdots, 2 n^{2} \\
\boldsymbol{\Upsilon}_{k \mid k-1, i}=\boldsymbol{h}\left(\boldsymbol{\chi}_{k \mid k-1, i}\right), \quad i=0,1, \cdots, 2 n^{2}
\end{gathered}
$$

Here, $\omega_{i}$ and $\chi_{k-1 \mid k-1, i}$ are obtained using the fifth-degree cubature rule and are given by

$$
\begin{aligned}
\omega_{i}=\left\{\begin{aligned}
\frac{2}{n+2} & i=0 \\
\frac{1}{(n+2)^{2}} & i=1,2, \cdots, 2 n(n-1) \\
\frac{4-n}{2(n+2)^{2}} & i=2 n(n-1)+1, \cdots 2 n^{2}
\end{aligned}\right. \\
\chi_{k-1 \mid k-1, i}=\hat{\boldsymbol{x}}_{k-1 \mid k-1}+\sqrt{\boldsymbol{P}_{k-1 \mid k-1}} \xi_{i}, \\
i=0,1, \cdots, 2 n^{2}
\end{aligned}
$$

where,

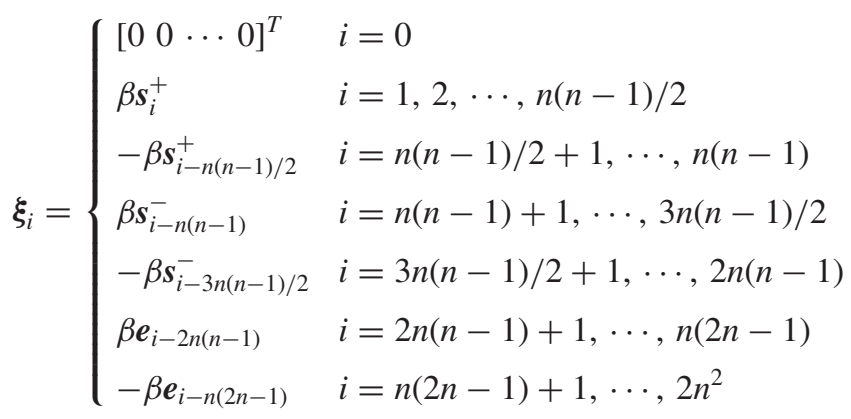

The parameter $\boldsymbol{s}_{j}^{+}$and $\boldsymbol{s}_{j}^{-}$are given by

$$
\left\{\begin{array}{cl}
\boldsymbol{s}_{j}^{+}=\sqrt{1 / 2}\left(\boldsymbol{e}_{p}+\boldsymbol{e}_{q}\right) & p<q ; p, q=1,2, \cdots, n \\
\boldsymbol{s}_{j}^{-}=\sqrt{1 / 2}\left(\boldsymbol{e}_{p}-\boldsymbol{e}_{q}\right) & p<q ; p, q=1,2, \cdots, n
\end{array}\right.
$$

where, $\boldsymbol{e}_{i}$ is the unit vector in $\mathbf{R}^{n}$ with the $i$ th element being 1, the parameter $\beta=\sqrt{n+2}$, and $j$ is given by

$$
\begin{cases}j=1 & q=2, p=1 \\ j=\frac{(q-1)(q-2)}{2}+p & q>2, p<q\end{cases}
$$

To embed the fifth-degree cubature rule into the QEKF algorithm, measurement Eqs. (28) and (29) need to be rewritten. According to the solution for the statistical linear regression of the nonlinear measurement equation, ${ }^{32)}$ the measurement matrix $\boldsymbol{H}_{k}$ is given by

$$
\boldsymbol{H}_{k}=\boldsymbol{P}_{x y, k \mid k-1}^{T} \cdot\left(\boldsymbol{P}_{k \mid k-1}\right)^{-1}
$$

where, $\boldsymbol{P}_{x y, k \mid k-1}$ is the cross-covariance

$\boldsymbol{P}_{x y, k \mid k-1}=\sum_{i=0}^{2 n^{2}} \omega_{i}\left(\boldsymbol{\chi}_{k \mid k-1, i}-\hat{\boldsymbol{x}}_{k \mid k-1}\right)\left(\boldsymbol{\Upsilon}_{k \mid k-1, i}-\hat{\boldsymbol{y}}_{k \mid k-1}\right)^{T}$

According to Eqs. (28) and (56), it is easy to obtain that $\boldsymbol{P}_{y y, k \mid k-1} \triangleq \boldsymbol{S}_{k}$

$$
=\sum_{i=0}^{2 n^{2}} \omega_{i}\left(\boldsymbol{\Upsilon}_{k \mid k-1, i}-\hat{\boldsymbol{y}}_{k \mid k-1}\right)\left(\boldsymbol{\Upsilon}_{k \mid k-1, i}-\hat{\boldsymbol{y}}_{k \mid k-1}\right)^{T}+\boldsymbol{R}_{k}
$$

Substitute Eqs. (64) and (66) into Eq. (29), and the gain matrix $\boldsymbol{K}_{k}$ is rewritten as follows

$$
\boldsymbol{K}_{k}=\boldsymbol{P}_{x y, k \mid k-1}\left(\boldsymbol{P}_{y y, k \mid k-1}\right)^{-1}
$$

Therefore, Eqs. (66) and (67), and Eqs. (32), (37) and (38) compose the measurement update of QHCKF. Note that $E\left(\boldsymbol{y}_{k} \mid \boldsymbol{Y}_{k-1}^{q}, \boldsymbol{y}_{k} \in A_{i}\right)$ and $\operatorname{cov}\left(\boldsymbol{y}_{k} \mid \boldsymbol{Y}_{k-1}^{q}, \boldsymbol{y}_{k} \in A_{i}\right)$ in Eqs. (32) and (37) can be calculated as that in QEKF.

\section{Simulation}

In this section, numerical simulation studies are performed to verify the effectiveness of the proposed QEKF and QHCKF methods for target tracking in the boost stage using quantized measurements of SBIRCs. Two GEO satellites are used for tracking the target. The measurements of two satellites are stacked into a four-dimensional vector.

\subsection{Simulation scene and simulation conditions}

The target trajectory in the boost phase is simulated in ECEF-CS as shown in Fig. 5, and the simulation parameters are given in Table 2 .

\subsection{Simulation results}

We compare the proposed QHCKF and QEKF methods and the original EKF (EKF-Q), the EKF with quantized measurement based on the AUN assumption (EKFAUN $),{ }^{14)}$ the approximate MMSE filtering algorithm (NMMSE), ${ }^{15)}$ and the EKF with pure Gauss measurements (EKF-G). For each method, we ran 100 Monte Carlo simulations and obtained the time history of RMSE of the position 


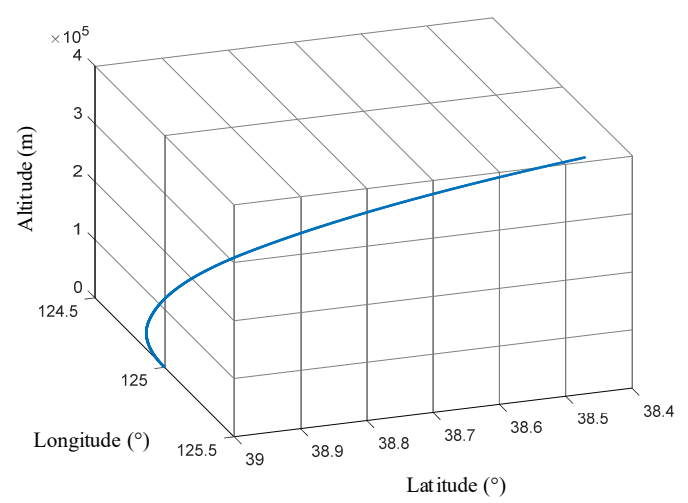

Fig. 5. Target trajectory in the boost phase.

Table 2. Simulation parameters.

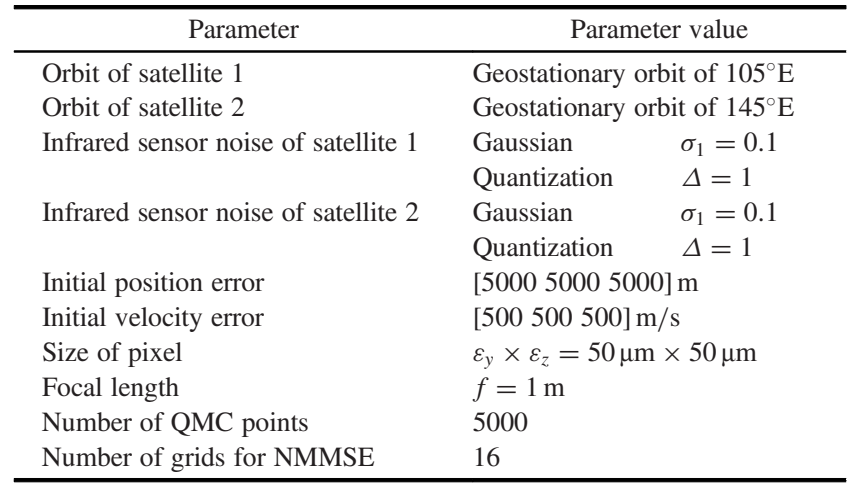

and velocity estimates (see Figs. 6 and 7). The RMSE for time step $k$ is calculated by

$$
\operatorname{RMSE}_{k}=\sqrt{\frac{1}{M} \sum_{i=1}^{M}\left(\hat{x}_{k}^{i}-x_{k}\right)^{2}}
$$

where, $M$ denotes the number of Monte Carlo simulations (i.e., 100 times), $\hat{x}_{k}^{i}$ denotes the estimated state at time $k$ in the $i$ th run, and $x_{k}$ denotes the true state at time $k$.

Figures 6 and 7 show the RMSEs from 100 Monte Carlo simulations for position and velocity at every time instance, respectively. Furthermore, the average position and velocity RMSEs during 10-60s for five algorithms are given in Table 3. It can be seen that the EKF-Q algorithm performs the worst. EKF-AUN performs better than EKF-Q but worse than NMMSE, QEKF and QHCKF. This is because the AUN assumption does not include saturation nor correlation properties from quantization, which results in poor estimation performance. Comparing EKF-AUN, EKF-Q and NMMSE, the estimation accuracy of the proposed QHCKF and QEKF methods are closer to that of EKF-G, which demonstrates the effectiveness of QHCKF and QEKF in target tracking using quantized measurements. The QHCKF algorithm performs even better than QEKF, which means that QHCKF is more effective in dealing with nonlinear models than QEKF. In addition, under the same Monte Carlo simulations, the curve of the RMSEs of NMMSE, QEKF and QHCKF are smoother than those of EKF-Q and EKF-AUN, which means that

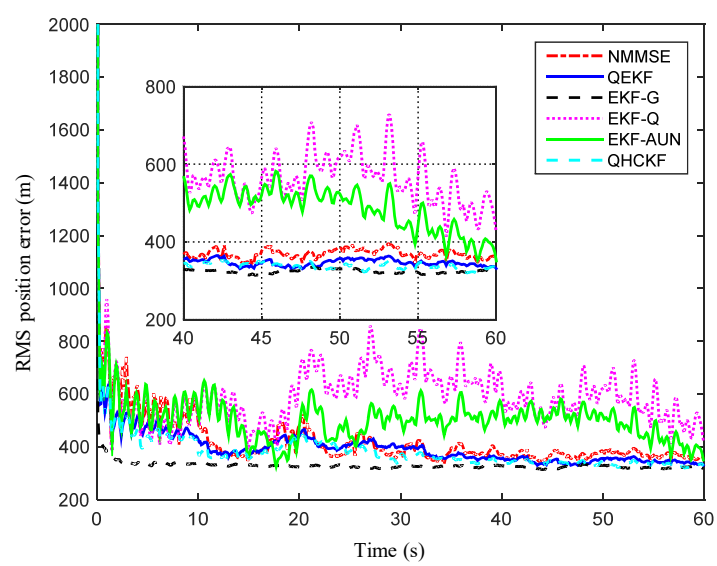

Fig. 6. RMS position errors of different algorithms.

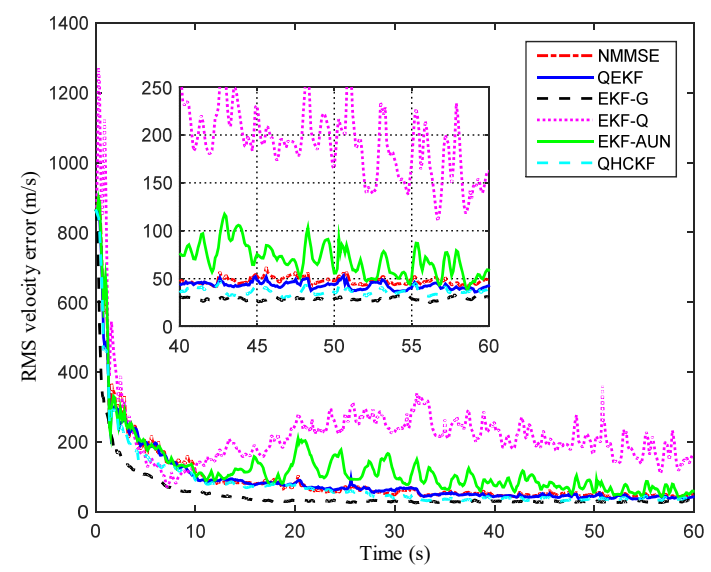

Fig. 7. RMS velocity errors of different algorithms.

Table 3. Average RMSEs during 10-60 s for five algorithms.

\begin{tabular}{lcccccc}
\hline & EKF-G & QEKF & QHCKF & NMMSE & EKF-AUN & EKF-Q \\
\hline Position (m) & 325.76 & 375.31 & 360.90 & 387.19 & 489.90 & 599.56 \\
Velocity (m/s) & 31.53 & 58.48 & 53.34 & 59.75 & 95.77 & 214.20 \\
\hline
\end{tabular}

NMMSE, QEKF and QHCKF eliminate more uncertainty than EKF-Q and EKF-AUN.

To investigate the effects of pixel size and the focal length on the algorithms, the algorithms with different sized pixels (i.e., $\varepsilon_{y}, \varepsilon_{z}$; note that the pixels are assumed to be square, $\left.\varepsilon_{y}=\varepsilon_{z}\right)$ and different focal lengths $(f)$ are compared. The mean RMSE of position and velocity with different pixel sizes and different focal lengths are shown in Figs. 8 and 9 , respectively.

It can be seen from Fig. 8 that performance improved when the pixel size decreases. Furthermore, the smaller the pixels, the closer the performance between different algorithms, which is not hard to explain because the smaller the pixels, the smaller the impact of quantization noise in the system, thereby eliminating the differences between different algorithms. In Fig. 9, the RMSEs of the algorithms with different focal lengths are given. It can be seen that the proposed QHCKF algorithm performed the best and performance improved when the focal length increased. 

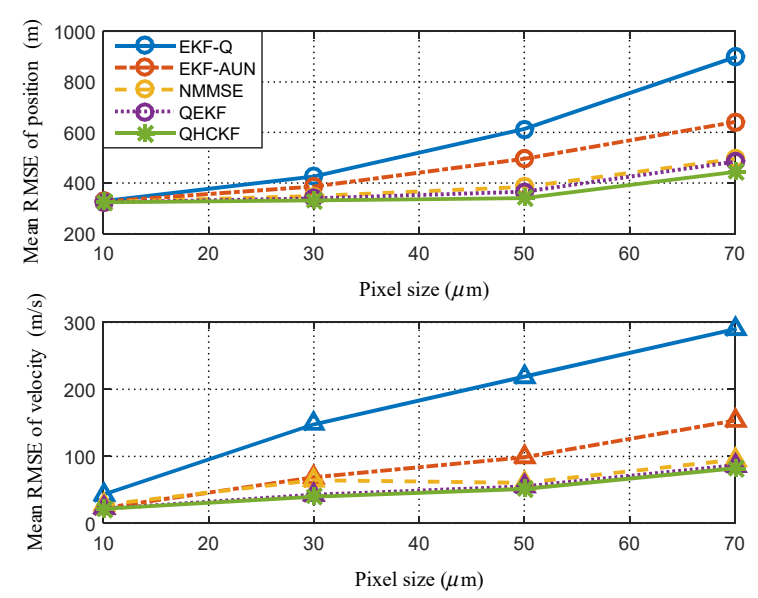

Fig. 8. RMSEs of the algorithms with different pixel sizes.
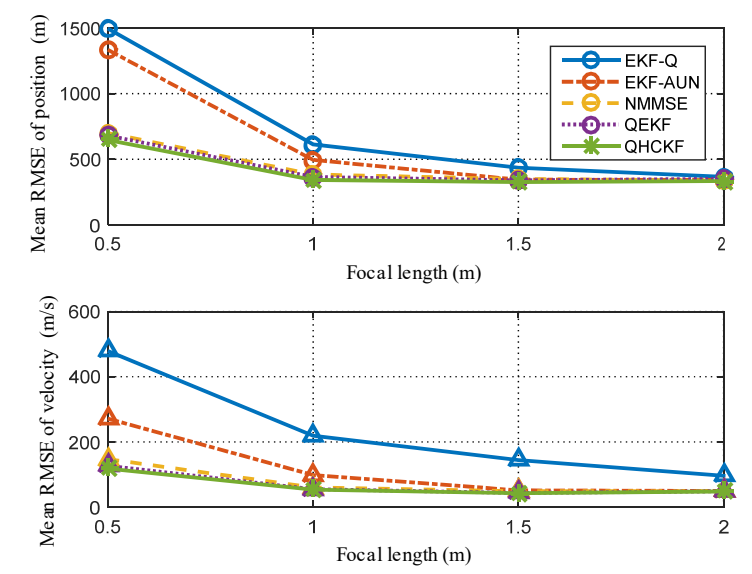

Fig. 9. RMSEs of the algorithms with different focal lengths.

In order to further compare the efficiency of NMMSE and the proposed QEKF algorithm, 100 Monte Carlo simulations were carried out for two algorithms under a different number of grids (i.e., NMMSE) and different integration points (i.e., QEKF). The average running time and corresponding RMSE are calculated and the relationship between them is shown in Fig. 10 and Fig. 11, respectively. It can be seen that NMMSE spends more time to obtain the same estimation accuracy as QEKF.

\section{Conclusion}

In this paper, the quantization noise model of SBIRCs is given using a mid-riser quantizer. Additionally, estimation algorithms with quantized measurements named QEKF and QHCKF are proposed based on the conditional mean estimate for target tracking in the boost stage. To calculate the multi-dimensional integrals in the derived measurement update, a numerical integration method is proposed by combining Genz's transformation and the QMC method. In simulations, the measurements are estimated based on the imaging principle of vision sensors. The simulation results show that the QHCKF and QEKF methods proposed outperform other algorithms in the presence of quantization noise and have the

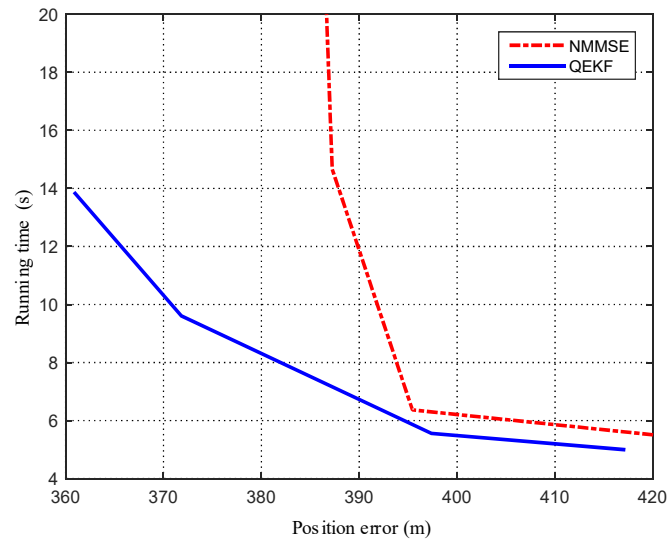

Fig. 10. The relationship between the running time and position error for two algorithms.

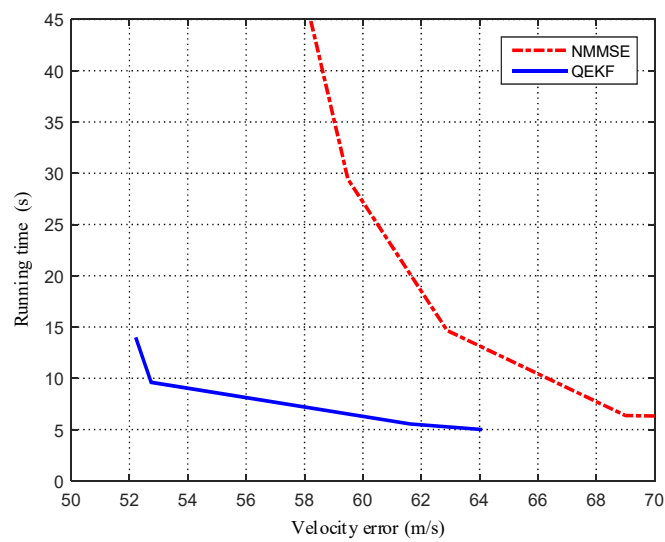

Fig. 11. The relationship between the running time and velocity error for two algorithms.

closest performance to that of pure Gauss noise. When the pixel size decreases and focal length increases, the tracking performance improves. In addition, simulation results show that the proposed QHCKF fifth-degree cubature rule embedded algorithm performs better than QEKF. Therefore, we claim that the model proposed is applicable for modeling measurements of vision sensors and that the QEKF and QHCKF methods are effective. Future work will focus on out-of-sequence measurements caused by communication and processing latency and real data experiments.

\section{References}

1) Rudd, J. G., Marsh, R. A., and Roecker, J. A.: Surveillance and Tracking of Ballistic Missile Launches, IBM J. Res. Dev., 38, 2 (1994), pp. 195-216.

2) Lu, Q., Bar-Shalom, Y., Willett, P., Ben-Dov, R., Milgrom, B., and Granström, K.: Tracking Initially Unresolved Thrusting Objects Using an Optical Sensor, IEEE Trans. Aerosp. Electron. Syst., 54, 2 (2018), pp. 794-807.

3) Zhao, W. and Huang, S.: Ballistic Target PHD Filter Based on Infrared Focal Plane Ambiguous Observation, Math. Probl. Eng., 2017, pp. 1-8.

4) Dai, H., Zhang, Y., Zhou, H., and Zhao, S.: Space-based Infrared Sensors of Space Target Imaging Effect Analysis, Fourth Seminar on Novel Optoelectronic Detection Technology and Application, Proceedings of SPIE, 2018, 10697, pp. 1-7. 
5) Wen, C., Cai, Y., Wen, C., and Xu, X.: Optimal Sequential Kalman Filtering with Cross-correlated Measurement Noises, Aerosp. Sci. Technol., 26, 1 (2013), pp. 153-159.

6) Pasqual, M. and DeLaurentis, D.: Measurement Fusion via Covariance Intersection for Ballistic Missile Tracking, Infotech@aerosp. 2012 Gard. Grove, Calif., 2012, pp. 1-11.

7) Jia, B., Pham, K. D., Blasch, E., Shen, D., Wang, Z., and Chen, G.: Cooperative Space Object Tracking Using Space-based Optical Sensors via Consensus-based Filters, IEEE Trans. Aerosp. Electron. Syst., 52, 4 (2016), pp. 1908-1936.

8) Wang, X., Qin, W., Bai, Y., and Cui, N.: Trajectory Estimation for Ballistic Missile in Boost Stage Using Robust Filtering, IET Radar Sonar Navig., 11, 3 (2017), pp. 513-519.

9) Kolitz, S. E.: Passive-sensor Data Fusion, Signal Data Process. Small Targets, 1991, 1481, pp. 329-340.

10) Oppenheim, A. V. and Schafer, R. W.: Digital Signal Processing, Prentice Hall, Englewood Cliffe, New Jersey, 1975, pp. 5-10.

11) Curry, R. E., Velde, W. E. V., and Potter, J. E.: Nonlinear Estimation with Quantized Measurements-PCM, Predictive Quantization, and Data Compression, IEEE Trans. Inf. Theory, 16, 2 (1970), pp. $152-161$.

12) Widrow, B., Kollár, I., and Liu, M. C.: Statistical Theory of Quantization, IEEE Trans. Instrum. Meas., 45, 2 (1996), pp. 353-361.

13) Asmar, D. M., Kochenderfer, M. J., and Chryssanthacopoulos, J. P.: Vertical State Estimation for Aircraft Collision Avoidance with Quantized Measurements, J. Guid. Control Dyn., 36, 6 (2013), pp. 1797-1802.

14) Karlsson, R. and Gustafsson, F.: Filtering and Estimation for Quantized Sensor Information, Linköping University, Sweden, 2005.

15) Duan, Z., Jilkov, V. P., and Li, X. R.: State Estimation with Quantized Measurements: Approximate MMSE Approach, Proceedings of the 11th International Conference on Information Fusion, 2008, pp. 1-6.

16) Siouris, G. M., Chen, G., and Wang, J.: Tracking an Incoming Ballistic Missile Using an Extended Interval Kalman Filter, IEEE Trans. Aerosp. Electron. Syst., 33, 1 (1997), pp. 232-240.

17) Leong, P. H., Arulampalam, S., Lamahewa, T. A., and Abhayapala, T. D.: A Gaussian-sum Based Cubature Kalman Filter for Bearings-Only Tracking, IEEE Trans. Aerosp. Electron. Syst., 49, 2 (2013), pp. 1161-1176.

18) Wang, X., Qin, W., and Cui, B.: Robust Trajectory Estimation in Ballistic Phase Using Out-of-Sequence High-degree Cubature Huber Based Filtering, Trans. Jpn. Soc. Aeronaut. Space Sci., 60, 3 (2017), pp. 164-170.

19) Wang, X., Qin, W., Cui, N., and Wang, Y.: Robust High-degree Cubature Information Filter and Its Application to Trajectory Estimation for Ballistic Missile, Proc. Inst. Mech. Eng. Part G J. Aerosp. Eng., 232, 12 (2018), pp. 2364-2377.

20) Yu, M., Oh, H., and Chen, W.: An Improved Multiple Model Particle Filtering Approach for Manoeuvring Target Tracking Using Airborne GMTI with Geographic Information, Aerosp. Sci. Technol., 52 (2016), pp. 62-69.

21) Ruan, Y., Willett, P., Marrs, A., Palmier, F., and Marano, S.: Practical Fusion of Quantized Measurements via Particle Filtering, IEEE Trans. Aerosp. Electron. Syst., 44, 1 (2008), pp. 15-29.

22) Shi, Z., Junbao, W., Ping, L., and Shouda, F.: Hierarchical Search Strategy in Particle Filter Framework to Track Infrared Target, Neural Comput. Appl., 29, 2 (2018), pp. 469-481.

23) Genz, A.: Numerical Computation of Multivariate Normal Probabilities, J. Comput. Graph. Stat., 1, 2 (1992), pp. 141-149.

24) Genz, A. and Bretz, F.: Comparison of Methods for the Computation of Multivariate $t$ Probabilities, J. Comput. Graph. Stat., 11, 4 (2002), pp. 950-971.

25) Yu, M., Chen, W., and Chambers, J.: State Dependent Multiple Modelbased Particle Filtering for Ballistic Missile Tracking in a Lowobservable Environment, Aerosp. Sci. Technol., 67 (2017), pp. 144-154.

26) Rodriguez, J. J. and Aggarwal, J. K.: Stochastic Analysis of Stereo Quantization Error, IEEE Trans. Pattern Anal. Mach. Intell., 12 (1990), pp. 467-470.

27) Karlsson, R. and Gustafsson, F.: Particle Filtering for Quantized Sensor Information, 13th Eur. Signal Process. Conf. EUSIPCO, 2005, pp. 1-6.

28) Widrow, B. and István, K.: Quantization Noise, Cambridge Univ. Press, New York, 2008, pp. 61-69.

29) Wang, X. and Hickernell, F. J.: Randomized Halton Sequences, Math. Comput. Model., 32, 7 (2000), pp. 887-899.

30) Bar-Shalom, Y., Li, X.-R., and Kirubarajan, T.: Estimation with Applications to Tracking and Navigation, Wiley, New York, 2001, pp. 381-385.

31) Haykin, S. and Arasaratnam, I.: Cubature Kalman Filters, IEEE Trans. Automat. Contr., 54, 6 (2009), pp. 1254-1269.

32) Lefebvre, T., Bruyninckx, H., and De Schutter, J.: Comment on 'A New Method for the Nonlinear Transformation of Means and Covariances in Filters and Estimators,' IEEE Trans. Automat. Contr., 47, 8 (2002), pp. 1406-1408. 